\title{
Laser-induced breakdown spectroscopy (LIBS)
}

\author{
Jagdish P. Singh • José R. Almirall • \\ Mohamad Sabsabi • Andrzej W. Miziolek
}

Published online: 11 May 2011

(C) Springer-Verlag 2011

Laser-induced breakdown spectroscopy (LIBS) is an emerging technique for materials analysis that is rapidly maturing and is becoming increasingly accepted as an important tool in analytical chemistry. LIBS is also advancing as a technology as new commercial instruments are becoming available. The core attributes of (1) real-time analysis; (2) no sample preparation; (3) high sensitivity; (4) high specificity for materials identification; (5) sensitivity to all chemical elements in each laser shot; as well as (6) uncommon versatility of point, standoff, as well as underwater-sensing provides a strong argument that LIBS will make a significant impact on science and society. A

This article was published in the special issue Laser-Induced Breakdown Spectroscopy with Guest Editors Jagdish P. Singh, Jose Almirall, Mohamad Sabsabi, and Andrzej Miziolek.

\section{J. P. Singh}

Institute for Clean Energy Technology,

Mississippi State University,

Starkville, MS 39759, USA

\section{J. R. Almirall $(\bowtie)$}

Department of Chemistry and Biochemistry and International Forensic Research Institute, Florida International University,

11200 SW 8th Street,

Miami, FL 33199, USA

e-mail: Almirall@fiu.edu

M. Sabsabi

National Research Council Canada, Industrial Materials Institute,

75 de Mortagne Blvd.,

Boucherville, Québec J4B 6Y4, Canada

A. W. Miziolek

US Army Research Laboratory, RDRL-WML-A,

Aberdeen Proving Ground, MD 21005-5069, USA bibliometric study of the LIBS literature shows clearly that the importance and the number of application areas related to LIBS and laser-based techniques continues to grow. The driving force for this growth appears to be its rapid and remote analysis capabilities for a wide variety of sample types, including the analysis where the requirement for little or no sample preparation is important and the consumption of very small amounts of the sample is critical. Additionally, the relative ease with which LIBS can be combined with other techniques, particularly molecular techniques such as Raman spectroscopy is an advantage. For proof of the impact that LIBS is already making, one needs to go no further than to learn about the next Mission to Mars scheduled for 2011/2012 where LIBS is the prime chemical analytical tool of choice.

This special issue on LIBS presents the latest progress in this rapidly evolving spectroscopic technique. The 18 articles represent a good balance between fundamental research on the LIBS phenomenology and the applied use of this technique. The papers presented indicate to the reader the active areas in the LIBS field. For example, research is focused on improving the sensitivity of the technique shows that the approach of double-pulse is still of interest. The understanding of physical phenomenon at the early stage of the plasma or the comparison between single- and double-pulse is still attracting further research. While Nd:YAG lasers operating at the fundamental wavelength $1,064 \mathrm{~nm}$ or its harmonics are most used for the laser-induced plasma generation in LIBS applications; some papers are focused on the use of the $\mathrm{CO}_{2}$ laser at $10.6 \mu \mathrm{m}$. In some cases, the use of this infrared laser may present benefits which can be further exploited. The analysis of slurries is a field of application where LIBS can offer a powerful tool for real-time analysis as the current analytical approaches in this field by conventional 
techniques are hampered in practice by their off-line character. In this special issue, certain recurring themes are well-represented including examples of quantitative analysis as well as the use of advanced chemometric models for spectral data analysis. LIBS continues to be applied for difficult analytical challenges including powder, residue, and food pathogen analysis. Important new areas are discussed including the use of LIBS for forensics applications as well as for determining the source area of natural materials.

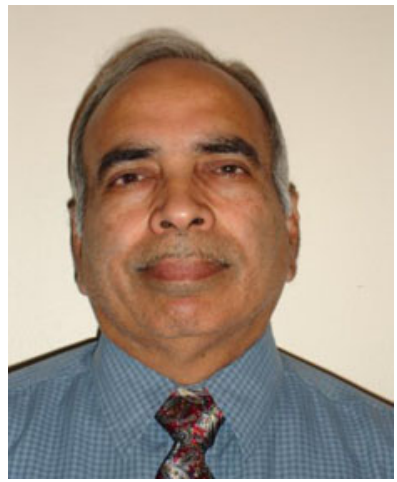

Jagdish P. Singh

is a Research Professor and Associate Director-Laser Diagnostics of Institute for Clean Energy Technology, Mississippi State University. He received his MS and Ph.D. degree in Physics from Banaras Hindu University, India, in 1974 and 1980. He was a Post-doctoral Research Associate at McMaster University, Canada, and Kansas State University. Prof. Singh has authored one book on LIBS and over 150 peer-reviewed scientific publications presented over 160 papers and supervised over $20 \mathrm{PhD}$ and MS students in Applied Physics. He is a Fellow of OSA and LASSI. His research interest includes Laser Spectroscopy, Optical Fiber Sensors, Laser Diagnostics for Combustion, and Hazardous Waste Management.

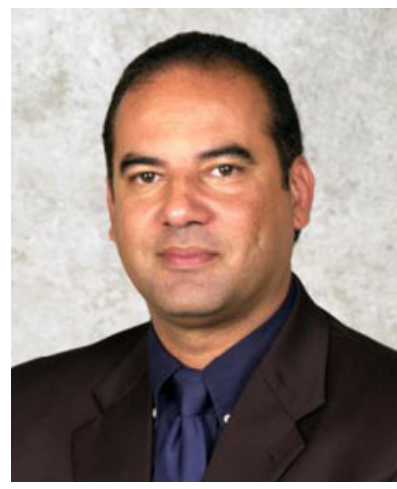

José R. Almirall

is a Professor in the Department of Chemistry and Biochemistry and Director of the International Forensic Research Institute at Florida International University. $\mathrm{He}$ was a practicing forensic scientist at the Miami-Dade Police Department Crime Laboratory for 12 years where he testified in over 100 criminal cases in state and federal courts prior to his academic appointment at FIU in 1998. Professor Almirall has authored one book and over 90 peer-reviewed scientific publications in the field of analytical and forensic chemistry, presented over 400 papers and workshops, and mentored over $30 \mathrm{PhD}$ and $\mathrm{MS}$ students in forensic chemistry research. His research interests include the development of analytical chemistry tools such as LIBS for use in forensic science and improvements in the value of trace evidence, in particular.

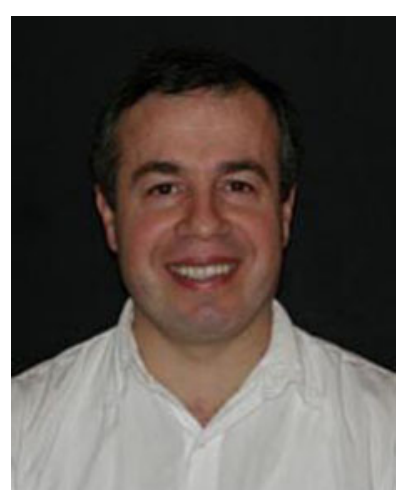

\section{Mohamad Sabsabi}

is the leader of the Optical Diagnostics Group at the National Research Council of Canada (NRCC) in Boucherville, Canada. His research interests include the industrial exploitation of laserbased spectroscopic techniques such as LIBS, LIFS, RELIBS, and LA, and modeling of the micro-plasma generated by laser ablation. The R\&D activities range from fundamental studies, via the development of prototype devices up to the transfer of laser measurement systems into industrial applications for online inspection tasks. He holds 11 patents and has published more than 330 scientific papers in refereed international journals, conference proceedings, conferences, reports, and contributions to two books, covering fundamental aspects and industrial applications of the laser-induced plasma.

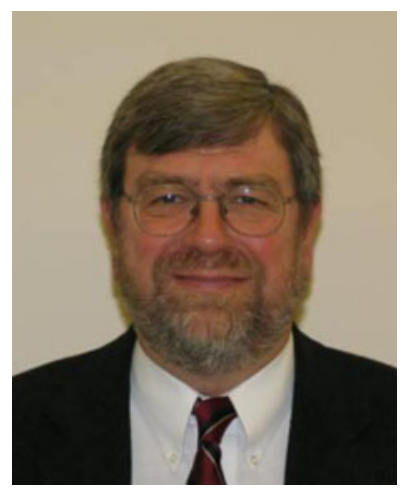

Andrzej W. Miziolek

is a senior Research Physicist at the US Army Research Laboratory. He has led the application of LIBS to the security and protection areas, as well as fostered the development of new technology (broadband high-resolution spectrometer, microwave enhancement) and the adaptation of chemometrics for spectral data processing. $\mathrm{He}$ has organized a number of conferences including the LIBS 2002 (2nd International) Conference and he founded the NASLIBS (North American Symposium on LIBS) series. He holds a Ph.D. from the University of California, Berkeley, is a Fellow of the Optical Society of America, and has published three books including "LIBS, Fundamentals and Applications," A.W. Miziolek, V. Palleschi, and I. Schechter, eds., Cambridge University Press, 2006. 